九州大学学術情報リポジトリ

Kyushu University Institutional Repository

\title{
APPLICATION OF KITAGAWA'S FUNCTIONAL INTEGRAL TO SOLUTIONS OF NON-LINEAR INTEGRAL EQUATIONS OF TWO VARIABLES
}

Strait, Peggy

Queens College of The City University of New York

https://doi.org/10.5109/13052

出版情報: 統計数理研究. 14 (3/4), pp. 57-60，1971-03. Research Association of Statistical Sciences

バージョン :

権利関係 : 


\title{
APPLICATION OF KITAGAWA'S FUNCTIONAL INTEGRAL TO SOLUTIONS OF NON-LINEAR INTEGRAL EQUATIONS OF TWO VARIABLES
}

\author{
By
}

\section{Peggy Stralt*}

(Received January 15, 1971)

\section{Introduction}

R. H. Cameron and W. T. Martin derived an expression [1], in terms of Wiener integrals, for the solution of a class of non-linear integral equations of a single variable. This note shows that by employing a lemma of J.D. Kuelbs [3], the result of Cameron and Martin may be easily extended (Theorem 1) to an expression for the solution of non-linear integral equations of two variables. The extended expression is in terms of the integral over the space of continuous functions of two voriables defined by T. Kitagawa [2], and extended by J. Yeh [7]. The essential properties of the Wiener integral (over 1-dimensional space) required for the proof given by Cameron and Martin are a Fubini theorem and a lemma concerning the Wiener measure of functions of one varioble in a small neighborhood. This note shows that both of these properties are also possessed by the integral over the space of continuous functions of two variables.

\section{Extended Kitagawa Integral in Function Space of Two Variables}

Let $C_{2}$ be the collection of continuous functions $\{x(t, \tau)\}$ on the unit square $0 \leqq t, \tau \leqq 1$ satisfying $x(0, \tau)=x(t, 0)=0$. Integration on this space of functionals of the type $H\left[x\left(t_{1}, \tau_{1}\right), \cdots x\left(t_{r}, \tau_{s}\right)\right.$ where $H\left[\eta_{11}, \cdots \eta_{r s}\right]$ is a function of $r s$ real variables $\left\{\eta_{h k}\right\}, h=1,2, \cdots, r ; k=1,2, \cdots, s$; and $\left\{t_{h}\left\{,\left\{\tau_{k}\right\}\right.\right.$ are preassigned division points of the unit intervals $0 \leqq t \leqq 1,0 \leqq \tau \leqq 1$ satisfying $0=t_{0} \leqq t_{1} \leqq \cdots \leqq t_{r} \leqq t_{r+1}=1,0=\tau_{0} \leqq \tau_{1}$ $\leqq \cdots \tau_{s} \leqq \tau_{s+1}=1$ was defined by $T$. Kitagawa [2] to be

$$
\begin{aligned}
& \int_{C_{2}}^{w} H\left[x\left(t_{1}, \tau_{1}\right), \cdots x\left(t_{r}, \tau_{s}\right)\right] d_{w} x \\
& \quad=\int_{-\infty}^{\infty} \cdots \int_{-\infty}^{\infty} H\left[\eta_{11}, \cdots \eta_{r s}\right] \prod_{h=1}^{r} \prod_{k=1}^{s} p\left(\Delta_{h k}\right) d \eta_{11} \cdots d \eta_{r s}
\end{aligned}
$$

where

$$
p\left(\Delta_{h k}\right)=\left[\pi\left(t_{h}-t_{h-1}\right)\left(\tau_{k}-\tau_{k-1}\right)\right]^{-1} \exp \left\{-\frac{\left(\eta_{h, k}-\eta_{h, k-1}-\eta_{h-1, k}+\eta_{h-1, k-1}\right)^{2}}{\left(t_{h}-t_{h-1}\right)\left(\tau_{k}-\tau_{k-1}\right)}\right\}
$$

with the understanding that $\eta_{0, j}=\eta_{i, 0}=0$.

* Queens College of The City University of New York. 
J. Yeh showed [7] that this integration is with respect to a probability measure $w$ on an interval class (Boolean algebra of sets) in $C_{2}$ and that therefore the integral could be extended to more general functionals. The measure $w$ (also called Wiener measure in $C_{2}$ ) was defined as follows. Let $E$ be a Lebesgue measurable subset of the $r s$-dimensional Euclidean space $R_{r s}$. Let $\left\{t_{h}\right\},\left\{\tau_{k}\right\}$ and $p\left(\Delta_{h k}\right)$ be defined as above and let a subset (interval) $I$ of $C_{2}$ be defined as $I\left\{t_{1}, t_{2}, \cdots t_{r}, \tau_{1}, \cdots \tau_{s}, E\right\}=\left\{x \in C_{2}\right.$ : $\left.\left(x\left(t_{1}, \tau_{1}\right), \cdots x\left(t_{r}, \tau_{s}\right)\right) \in E\right\}$. Then the Wiener measure of $I$ is defined as

$$
w(I)=\int_{E}^{(r s)} \cdots \int \prod_{h=1}^{i} \prod_{k=1}^{s} p\left(\Delta_{h k}\right) d \eta_{11} \cdots d \eta_{r s} .
$$

The collection $\Im$ of all sets of the form $I$ was shown to be an algebra of sets and $w$ a probability measure on that algebra. Thus there is a whole class of functionals $\Phi(x)$ which are integrable over the space $C_{2}$ with respect to this measure and we denote the integral by $\int_{C_{2}}^{w} \Phi(x) d_{w} x$. Also, as in the case of Wiener integrals over the space of functions of one variable, if $\Phi(x)$ is integrable and $S$ is a measurable subset of $C_{2}$, we define

$$
\int_{S}^{w} \Phi(x) d_{w} x=\int_{c_{2}}^{w} \phi(x) d_{w} x
$$

where

$$
\phi(x)= \begin{cases}\Phi(x) & \text { for } x \text { in } S \\ 0 & \text { otherwise }\end{cases}
$$

\section{Fubini Theorem for Extended Kitagawa Integrals in $C_{2}$}

Cameron and Martin stated in [1] that the Fubini theorem holds for two Wiener integrals or for Wiener and Lebesgue integrals since the Wiener mapping takes function space into a linear interval to which the ordinary Fubini theorem applies. Although we can show in exactly the same manner (i. e. by means of a mapping to $C_{2}$ into the unit square) that the Fubini theorem holds for extended Kitagawa integrals over the space $C_{2}$, we need not use this method since we already know from section 2 that $w$ is a finite measure in $C_{2}$. This implies that the Fubini theorem holds for two Kitagawa integrals over $C_{2}$ or for Kitagawa integral over $C_{2}$ and Lebesgue integral over the unit square.

\section{Wiener Measure of Functions in Small Neighborhoods}

LEMMA 1. For each $x_{0}(t, \tau)$ in $C_{2}$ and each $\eta>0$ the set $T_{\pi}$ consisting of all functions $x(t, \tau)$ in $C_{2}$ satisfying

$$
\int_{0}^{1} \int_{0}^{1}\left\{x(t, \tau)-x_{0}(t, \tau)\right\}^{2} d t d \tau<\eta
$$

has positive Wiener measure

$$
\int_{T \eta}^{w} d_{w} x>0
$$


Proof. Lemma 3, page 358, of Kuelbs paper [3] states that if $E$ is an open subset of $C_{2}$, then $w(E)>0$. Observe that

$$
\left\{x: \int_{0}^{1} \int_{0}^{1}\left\{x(t, \tau)-x_{0}(t, \tau)\right\}^{2} d t d \tau<\eta\right\} \supseteqq\left\{x: \sup _{\substack{0 \leqq t \leq 1 \\ 0 \leqq \tau \leqq 1}}\left|x-x_{0}\right|<\sqrt{\eta}\right\}
$$

The set on the right of the inequality is open. Thus,

$$
\int_{T r}^{w} d_{w} x>0
$$

\section{An Expression for the Solution of Non-Linear Integral Equations of Two Variables}

THEOREM 1. Let $G(t, \tau, \xi, \eta, u)$ be continuous in $0 \leqq t \leqq 1,0 \leqq \tau \leqq 1,0 \leqq \xi \leqq 1$, $0 \leqq \eta \leqq 1,-\infty<u<\infty$ and let it satisfy there the uniform Lipschitz condition

$$
\left|G\left(t, \tau, \xi, \eta_{i}, u_{2}\right)-G\left(t, \tau, \xi, \eta, u_{1}\right)\right|<M\left|u_{2}-u_{1}\right| .
$$

Then if $y(t, \tau)$ is any continuous function in $0 \leqq t \leqq 1,0 \leqq \tau \leqq 1$ and vanishing at $t=0$ and at $\tau=0$, the integral equation $x(t, \tau)=y(t, \tau)+\int_{0}^{t} \int_{0}^{\tau} G(t, \tau, \xi, \eta, x(\xi, \eta)) d \xi d \eta$ has a unique solution $x_{0}(t$,$) given by$

$x_{0}(s, \sigma)=\lim _{\rho \rightarrow \infty} \frac{\int_{C_{2}}^{w} \exp \left[-\rho \int_{0}^{1} \int_{0}^{1}\left\{y(t, \tau)-x(t, \tau)+\int_{0}^{t} \int_{0}^{t} G(t, \tau, \xi, \eta, x(\xi, \eta) d \xi d \eta\}^{2} d t d \tau\right] x(s, \sigma) d_{w} x\right.}{\int_{C_{2}}^{w} \exp \left[-\rho \int_{0}^{1} \int_{0}^{1}\left\{y(t, \tau)-x(t, \tau)+\int_{0}^{t} \int_{0}^{\tau} G(t, \tau, \xi, \eta, x(\xi, \eta) d \xi d \eta\}^{2} d t d \tau\right] d_{w} x\right.}$

where the lim is taken in the $L_{2}$ sense for ordinary Lebesque integrals, $0 \leqq s \leqq 1,0 \leqq \sigma$ $\leqq 1$, and the two integrals $\int_{c_{2}}^{w}$ are extended Kitagawa integrals as defined in section 2 above.

This theorem is a direct generalization of Theorem 1 in [1]. We shall not include here a detailed proof because now that it has been ascertained in sections 2 through 4 that the essential properties of the Wiener integral required for the proof given by Cameron and Martin are also possessed by the extended Kitagawa integral over $C_{2}$, a proof for the two variable case may be obtained by referring to the original proof in [1]. One should begin with Section 3, The General Theorem, on page 285 of $[1]$, and continue through section 7 which concludes at the top of page 294.

As a final remark it should be noted that all results here are valid for the case of $n$ variables.

\section{References}

[1] R. H. Cameron and W. T. Martin, An expression for the solution of a class of non-linear integral eqnations, Amer. J. of Math., Vol. 66, (1944), pp. 281-298.

[2] T. Kitagawa, Analysis of variance applied to function spaces, Mem. Fac. Sci., Kyushu Univ., Ser. A, Vol. VI, No. 1 (1951), pp. 41-53. 
[3] J. Kuelbs, Additioe Functionals on $C(Y)$, Proceeding of the Amer. Math. Society, Vol. 19, No. 2, April, 1068, pp. 354-360.

[4] P. Strait, Sample function regularity for Gaussian process with the parameter in a Hilbert space, Pacific J. of Math., Vol. 19, No. 1, 1966, pp. 159-173.

[5] P. Strait, On Kitagawa's Functional Integral, Tohoku Math. Journal, The Second Series, Vol. 19, No. 1, pp. 75-78, Mar., 1967.

[6] N. Wiener, Generalized harmonic analysis, Acta Math., Vol. 55, (1930), pp. 117-258.

[7] J. Yeh, Wiener measure in a space of functions of two variables, Trans. of Amer. Math. Soc., Vol. 95, 1960, pp. 433-450. 